\title{
Mothers Exaggerate Their Finger Movements While Demonstrating Object Manipulation to Their Infants
}

\author{
Kaori Nagata1, Eriko Yamamoto', Goh Matsuda², Kazuo Hiraki1 \\ ${ }^{1}$ Graduate School of Arts and Sciences, The University of Tokyo, Tokyo, Japan \\ ${ }^{2}$ General Medicine and Medical Education, Kyoto Prefectural University of Medicine, Kyoto, Japan \\ Email: nagata.kaori@ardbeg.c.u-tokyo.ac.jp
}

How to cite this paper: Nagata, K., Yamamoto, E., Matsuda, G., \& Hiraki, K. (2018). Mothers Exaggerate Their Finger Movements While Demonstrating Object Manipulation to Their Infants. Psychology, 9, 2609-2624.

https://doi.org/10.4236/psych.2018.912149

Received: October 17, 2018

Accepted: November 9, 2018

Published: November 12, 2018

Copyright $\odot 2018$ by authors and Scientific Research Publishing Inc. This work is licensed under the Creative Commons Attribution International License (CC BY 4.0).

http://creativecommons.org/licenses/by/4.0/

\begin{abstract}
We investigated whether mothers exaggerate the fine movements of their fingers when interacting with their infants, and whether infant-directed action is influenced by mothers' beliefs about a demonstration-observer's knowledge. Fifteen mothers demonstrated how to use a novel toy to their infant (Infant condition), an adult family member (Uninformed Adult condition), and an adult female who already knew how to use the toy (Informed Adult condition). An optical motion capture system was used to examine the mother's wrist and finger movements, and her gaze was video recorded. Compared with the Uninformed Adult condition, in the Infant condition, mothers exaggerated their wrist movements when holding the toy in the same way as in previous studies, opened their fingers wider when reaching for the toy, and looked at the observer more often. There was no significant difference in hand movements between the Informed Adult and Uninformed Adult conditions: that is, the observer's level of prior knowledge did not affect the mothers' motions. This suggests that mothers may exaggerate their finger movements in order to attract their infants' attention, not only while holding the object but even while initially reaching for it, while also monitoring the infant.
\end{abstract}

\section{Keywords}

Mother, Infant, Interaction, Infant-Directed Action, Hand Movements

\section{Introduction}

When interacting with infants, mothers' actions become exaggerated, slow, and more repetitive (e.g., Brand, Baldwin, \& Ashburn, 2002; Fukuyama et al., 2015; 
Rohlfing, Fritsch, Wrede, \& Jungmann, 2006), a phenomenon referred to as "infant-directed action". Initially, the characteristics of infant-directed action were identified using a manual coding system (Brand et al., 2002), in which human coders estimate mothers' demonstration according to a single global rating. Later, infant-directed action began to be investigated by examining the kinematics of relatively large joints, such as their wrists and elbows using a three-dimensional (3D) motion tracking system (e.g., Fukuyama et al., 2015; Nagai, Nakatani, \& Asada, 2010, Rohlfing et al., 2006). However, this technique is insufficient to characterize infant-directed action fully, because most object manipulation in everyday life is carried out using movement of the fingers.

Previous studies of object manipulation in adults show that adults' finger movements are modulated by social context. For example, the distance between the index finger and thumb (peak grip aperture) used to grasp an object increases when an individual is interacting with a partner (Becchio, Sartori, Bulgheroni, \& Castiello, 2008a, 2008b; Sacheli, Candidi, Pavone, Tidoni, \& Aglioti, 2012; Sartori, Becchio, Bara, \& Castiello, 2009). Therefore, it might be expected that, when interacting with infants, mothers exaggerate not only the movements of their large joints, but also their finger movements. To investigate this, it is necessary to examine the precise movements of the fingers during demonstrations of object manipulation.

Furthermore, it is not clear why mothers exaggerate their motions when interacting with infants. Infant-directed action is observed by comparing a mother's demonstrations of an action to her infant and to an adult (e.g., Brand et al., 2002). However, there are many differences between infants and adults; therefore, there are several factors that may explain the emergence of infant-directed action. First, one possible relevant factor may be the notion of a "baby schema", as proposed by Lorentz (1965). Lorentz suggests that infants' physical features, such as their round faces, large heads, and large eyes, capture adults' attention and motivate adults' caretaking behavior toward their infants. In this vein, Glocker et al. (2009) report that photographs of infants with a high-intensity baby schema elicit a stronger motivation for caretaking in adult participants than do photographs of infants with a low-intensity baby schema. According to previous studies, infant-directed action attracts infants' attention (Brand \& Shallcross, 2008; Koterba \& Iverson, 2009). Additionally, infants imitate the use of a novel toy more successfully when they are shown how to use it with infant-directed action (Williamson \& Brand, 2014). Given this functionality of infant-directed action, the visual features of the baby schema might elicit infant-directed action from adults and thereby aid infants' development.

Second, infants' immature ability to attend might cause infant-directed action. Brand et al. (2002) show that mothers look longer at their infant than at an adult family member when demonstrating a novel toy manipulation. The authors suggest that this difference in the mother's gaze behavior is due to the infant's capacity for attention, and that the mother modifies her movements to enhance her infant's attention to her action. Indeed, mothers tend to display an object 
explicitly (e.g., by shaking it) to attract an infant's attention while focusing on the infant's face (Deák, Krasno, Triesch, Lewis, \& Sepeta, 2014).

However, the characteristics of infant-directed action are also observed in adult-adult interaction. When a communication partner uses a different language or a demonstrator feels that their partner has not understood an instruction well, the speaker uses more gestures to emphasize and complement their verbal expression (LaFrance \& Mayo, 1978). In addition, when gestures are used as a main communicative behavior, the demonstrator moves closer to their communication partner to attract his or her attention (Furuyama, 2000; Streeck, 1994). In the same way, it has been shown that English speakers tend to speak with clearer articulation when talking to second-language English learners (adult foreigners) than when talking to native English speakers (Uther, Knoll, \& Burnham, 2007). Adults and experts generally assist infants and beginners (Wood, Bruner, \& Ross, 1976). Thus, it might be not only an infant's visual features and immature perception that elicit infant-directed action, but also the observer's level of knowledge about a demonstration. However, no study has investigated whether characteristics of infant-directed action emerge when mothers give a demonstration to an adult who is unfamiliar with the demonstration, compared to their demonstration to an adult who is familiar with it.

Thus, the aims of our study were to investigate whether mothers' finger movements are exaggerated when they interact with their infants and whether an observer's lack of knowledge of the mother's demonstration causes characteristics of infant-directed action to emerge. Each participating mother demonstrated how to use a novel toy to three individuals: her own infant, who was unfamiliar with the toy; an adult family member who was unfamiliar with the toy; and a female adult who was familiar with the toy. The trajectories of the mother's wrist and finger movements were recorded with an optical motion capture system. To confirm the emergence of exaggerated action, mothers' demonstrations to their infants were compared to their demonstrations to adults who were unfamiliar with the toy. Additionally, to examine the appearance of characteristics of infant-directed action related to the observer's knowledge of the toy manipulation, we compared the mothers' demonstrations to adults who were unfamiliar with the toy and to an adult who was familiar with it. Furthermore, we analyzed mothers' gaze behavior during demonstration.

We hypothesized that mothers' wrist and finger movements would become larger when they demonstrated to their infants compared to when they demonstrated to the uninformed adult, in the same way as observed in previous studies of reach-to-grasp action in social interaction. In addition, we expected that mothers would look longer at their infants than at the uninformed adult during their demonstrations. Concerning the association between mothers' behavior and the observer's knowledge about the subject of the demonstration, we hypothesized that larger motions would be observed when mothers demonstrated to an uninformed adult compared to an informed adult, in line with previous studies of gesture. 


\section{Methods}

\subsection{Participants}

Fifteen triads participated in this study, each consisting of a mother, her infant (8 males and 7 females), and an adult family member who was highly familiar to the mother ( 4 of their own mothers and 11 partners) (Table 1). One additional triad was excluded because the mother did not demonstrate the required task to the observers. The sample size was determined to produce an effect size of $d=$ 0.80 by using a power analysis with a power level of 0.90 . We confirmed in a pilot test that infants at this age could correctly manipulate the toy we used after receiving their mothers' instructions. We asked the mothers to bring a highly familiar adult with them to participate, as in Brand et al. (2002), since differences in intimacy across conditions might affect the fluidity of mothers' demonstrations. All mothers were right-handed, as assessed by the Edinburgh Handedness Inventory (Oldfield, 1971). Prior to their participation, the experimental procedure was explained to all participants, and they gave written informed consent. Participants were recruited through birth records at a city hall branch office, and parents who expressed interest in enrolling their infants in the study were contacted via e-mail. This study conformed to the guidelines laid out under the Declaration of Helsinki and was approved by the Ethics Committee of the Graduate School of Arts and Science at the University of Tokyo.

\subsection{Apparatus}

This experiment was conducted in two separate rooms: a demonstrator room and an observer room. To control for the mother's postural position and visual information available to the observer, such as the appearance of the toy, we used two real-time TV monitors so that the mother and observer could see and interact with one another from these separate rooms during demonstrations (Figure 1(a) \& Figure 1(b)). The mother and observer could see each other through two 32-inch color TV monitors (TH-L32DT3, Panasonic, Inc., Osaka, Japan; LC-32E5, SHARP, Inc., Osaka, Japan) reflected onto a one-way mirror (H: $25 \mathrm{~cm} \times \mathrm{W}: 70$ $\mathrm{cm}$ ). A digital video camera (HXR-MC1, SONY, Inc., Tokyo, Japan), recording at a sampling rate of $30 \mathrm{fps}$, was placed behind each mirror to film the mother and observer so that they could make eye contact with each other. The mother

Table 1. Mean values (with standard deviation) of participants' data ${ }^{\mathrm{a}}$.

\begin{tabular}{lcc}
\hline & \multicolumn{1}{c}{ Age } & Range \\
\hline Mother & $35.5 \pm 4.5$ years & $28-46$ years \\
Infant & $29.1 \pm 3.2$ months & $24-34$ months \\
Adult family member & & \\
$\quad$ & & \\
$\quad$ Own mother & $63.7 \pm 5.1$ years & $55-67$ years \\
$\quad$ Partner & $34.5 \pm 5.6$ years & $24-46$ years \\
\hline
\end{tabular}

${ }^{\mathrm{a}}$ Values are mean $\pm \mathrm{SD}$. 


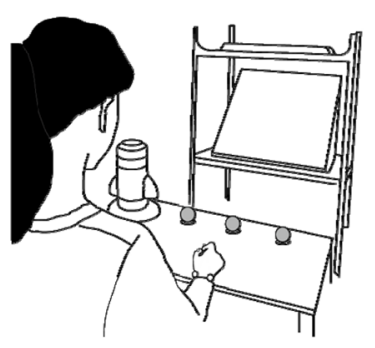

(a)

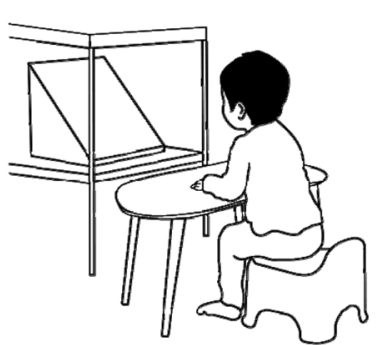

(b)

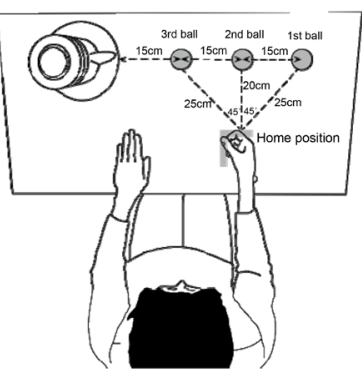

(c)

Figure 1. Experimental set-up of the demonstrator room (a), observer room (b), and toy used in the demonstration by mothers (c). Note: Figure 1 denotes an example of scene in this study. Source: This figure is our original creation.

sat in front of a table $(\mathrm{H}: 60 \mathrm{~cm} \times \mathrm{W}: 45 \mathrm{~cm} \times \mathrm{L}: 91 \mathrm{~cm})$ and faced the TV monitor, which was located approximately $1.5 \mathrm{~m}$ away, at eye level. To prevent changes to the mother's movements in response to the observer's voice, she could not hear the observer's voice from the demonstrator room, but the observer could hear the mother's voice through a microphone.

The trajectories of the mother's hand movements were recorded at a sampling rate of 300 Hzusing an optical motion capture system with seven cameras (OQUS300, Qualysis, Inc., Göteborg, Sweden). Infrared reflective markers were taped to the fingernail of the mother's index finger and thumb, and her right wrist (finger markers' diameter $=0.5 \mathrm{~cm}$, wrist marker's diameter $=1.5 \mathrm{~cm}$ ). The motion capture cameras and video camera were synchronized with an LED signal that was sent to these cameras at the same time.

The mother was asked to demonstrate how to use a novel toy (rocket ball, Combi, Inc., Tokyo, Japan) whose use included making a grasping action. This toy consisted of three balls, $5 \mathrm{~cm}$ in diameter, and a rocket-shaped container, $24.5 \mathrm{~cm}$ in height with a $5.5 \mathrm{~cm}$ diameter hole on the top and another hole underneath. Balls inserted into the top hole could roll out from the bottom hole only when the rocket's wings were pressed down. The toy used in this study was novel for all participants.

\subsection{Demonstration}

When the demonstration began, the mother placed her right hand at a home position on the table, with her index finger and thumb touching each other. The home positions of the mother's hand, three balls, and the rocket were always the same (Figure 1(c)). The mother then grasped each of the three balls in turn and placed it into the top hole of the rocket, moving from right to left from her point of view. She was instructed to return her right hand to the home position each time she put a ball into the rocket. After inserting all the balls, the mother held the rocket's wings with both hands and pressed them down; then, the balls rolled out of the bottom hole of the rocket. When the demonstration ended, the mother returned her hands to the home position. In all conditions and on all trials, 
she was instructed to speak to the observer using the same words: "First", "Second", and "Third", when she manipulated each corresponding ball; the timing of these words was not specified.

To evaluate the utility of this demonstration for adults, we conducted a preliminary test to check whether nine additional adults (three men and six women, aged $26.0 \pm 4.0$ years) could carry out the same toy manipulation that was shown in the demonstration on sight of the toy and without watching the demonstration. None of them performed the same manipulation as in the demonstration within a $60 \mathrm{~s}$ test period.

\subsection{Procedure}

In a brief warm-up period to allow the mother, her infant, and the adult family member to acclimatize to the experimental setting, the mother was familiarized with the female adult who was already familiar with the toy (the observer in the Informed Adult condition) by means of chatting with her for about $20 \mathrm{~min}$. She was also told that the female adult was familiar with the toy. The same female adult played the role of the observer in the Informed Adult condition in all cases. Subsequently, the mother moved to the demonstrator room and sat in front of the TV monitor, then practiced giving the demonstration 10 times. Meanwhile, the experimenter gave the toy to the infant and conducted a baseline test for $60 \mathrm{~s}$ before the mother's demonstration. The mother could not watch the baseline test.

Next, the mother was asked to perform the demonstration in front of three people in turn: her own infant, who was unfamiliar with the toy (Infant condition); her adult family member who was unfamiliar with the toy (Uninformed Adult condition); and the female adult who was already familiar with the toy (Informed Adult condition). The demonstration was repeated three times in each condition, with a $\sim 1$-min interval between demonstrations. A short rest period ( $3 \mathrm{~min}$ ) separated each condition. The order of conditions was randomized across participants, with all six possible orders used with two or three mothers each.

At the start of each demonstration, the mother drew the observer's attention to her by saying, "Look at me", and established eye contact. Next, the mother began the demonstration whenever she chose. After all trials were complete in the Infant and Uninformed Adult conditions, the mother instructed the observer: "Play with it". Subsequently, to determine whether the mother's demonstration had been able to facilitate the observer's learning about how to use the toy, the experimenter gave the toy to the observer and conducted an imitation test for 60 s. The mother could not watch the imitation tests.

\subsection{Outcome Measures}

First, we divided each manipulation of the balls during the demonstration into two action phases (reach-to-grasp and lift-to-place) and examined whether ex- 
aggerated actions emerged in each action phase. Second, we analyzed how long the mother looked at the observer and other objects involved in the demonstration. Finally, we assessed the observers' baseline and imitation test scores.

Kinematics of mothers' hand movements. Three-dimensional position data recorded from three infrared reflective markers (affixed to the right index finger, thumb, and wrist) were filtered using a second-order Butterworth low-pass filter with a cut-off frequency of $10 \mathrm{~Hz}$. Single trial data were divided into three ball manipulation periods; moreover, each single ball manipulation was divided into two phases: reach-to-grasp, which was the time interval between the onset of the ball manipulation and when the demonstrator touched the ball, and lift-to-place, which was the interval between the next frame following the end of the reach-to-grasp phase and when the demonstrator released the ball at the top hole of the toy (Supplementary Figure A1).

A previous study shows that the duration of mothers' demonstrations is longer with infants than with adults (Brand et al., 2002). Therefore, we computed the duration of the demonstrations by calculating the time between onset of the first ball manipulation and return of the mother's hands to the home position after she pressed down the rocket's wings. In addition, we analyzed phase duration, mean wrist velocity, and wrist trajectory for each of the two ball manipulation phases. These parameters were adopted from previous studies of infant-directed action (Fukuyama et al., 2015; Nagai et al., 2010; Rohlfing et al., 2006), in which infant-directed action has been found to be characterized by actions lasting longer, and occurring more slowly and in an exaggerated fashion. Additionally, we measured peak grip aperture in the reach-to-grasp phase and peak height of the wrist in both phases, because these indices have been shown to be sensitive to social context (Becchio et al., 2008a; Vesper, Schmitz, Safra, Sebanz, \& Knoblich, 2016). All kinematic analyses were performed using MATLAB 2007b (Math Works, Inc., MA, USA).

Mothers looking time. The mother's looking time during each of the two action phases was analyzed. Video recordings of the mother's gaze (30 fps) were analyzed frame-by-frame by a trained researcher to determine the target of her gaze and end of her gaze at this target, using the ELAN4.7.3 software (Lausberg \& Sloetjes, 2009). Gaze targets were coded into three categories: observer, ball, and rocket. Other actions, such as closing the eyes, were classified as "other". A second independent coder recoded a random $10 \%$ of all trials. There was a high level of agreement between the coders about gaze targets and corresponding looking times $(\kappa=0.93, p<0.01)$. Therefore, the data produced by the original coder were entered into analyses.

Baseline and imitation tests. Based on the video of each baseline and imitation test, the researcher evaluated whether the participant was successful in putting three balls into the top hole of the rocket using a binary rating: yes or no. The order of ball insertion was not considered in this coding. A second independent coder recoded a random $50 \%$ of participants' data from these trials. 
Perfect reliability in coding of the infants' and adult family members' actions was achieved $(\kappa=1.00)$. Therefore, the results revealed that none of the infants were successful in inserting the three balls into the top of the rocket in the baseline test. However, all infants and adult family members (uninformed adults) successfully inserted the three balls in the imitation test.

\subsection{Statistical Analyses}

We statistically analyzed each kinematic parameter using student's $t$ tests with Bonferroni correction to assess whether the appearance of exaggerated action was related to the observer's age (Infant vs. Uninformed Adult) or the observer's knowledge of how the toy should be used (Informed Adult vs. Uninformed Adult). Because there was no significant difference in kinematic parameters using family-wise error, the results shown in this study are uncorrected.

We analyzed looking time using a two-way repeated measures analysis of variance (ANOVA) over an angular transformation of the percentage value, in a 3 (condition: Infant vs. Uninformed Adult vs. Informed Adult) $\times 2$ (gaze target: observer vs. ball vs. rocket) design. If an interaction was significant $(p<0.05)$, post hoc comparisons using student's $t$ tests with Bonferroni correction were carried out.

Effect sizes were measured using $\eta_{p}^{2}$ (ANOVA) and Cohen's $d$ ( $t$ test). The significance level for all post hoc comparisons was set at (Bonferroni-corrected) $p<0.05$. All data are presented in the form mean \pm standard error (SE) across all participants. The results of all $t$ tests are reported in the Supplementary Material (Tables A1-A3). A Microsoft Excel file in supplementary material shows data sources for mothers' movements and looking time.

\section{Results}

We successfully obtained data from all 15 mothers, and we analyzed data from 15 mothers $\times 3$ trials $\times 3$ ball manipulations $=135$ ball manipulations in total for each condition.

\subsection{Kinematics of Mothers' Movements}

Table 2 shows mean values for all kinematic parameters. The demonstrations lasted significantly longer in the Infant condition than in the Uninformed Adult condition $(p<0.05)$.

In the reach-to-grasp phase, peak grip aperture was significantly larger in the Infant condition than in the Uninformed Adult condition $(p<0.05)$.

Regarding the lift-to-place phase, the duration of this phase was significantly longer in the Infant condition than in the Uninformed Adult condition $(p<$ $0.05)$. Furthermore, we observed that mean wrist velocity was significantly slower in the Infant condition than in the Uninformed Adult condition $(p<0.05)$. Wrist trajectory distances were significantly longer in the Infant condition than in the Uninformed Adult condition $(p<0.05)$. In addition, peak wrist heights 
Table 2. Mean values (with standard errors) of parameters describing the kinematics of mothers' movements .

\begin{tabular}{lccc}
\hline & Infant & $\begin{array}{c}\text { Uninformed } \\
\text { Adult }\end{array}$ & $\begin{array}{c}\text { Informed } \\
\text { Adult }\end{array}$ \\
\hline $\begin{array}{l}\text { Demonstration duration }(\mathrm{s})^{*} \\
\text { Reach-to-grasp phase }\end{array}$ & $16.90(0.90)$ & $14.70(0.82)$ & $14.45(0.88)$ \\
$\quad$ Phase duration $(\mathrm{s})$ & $1.02(0.03)$ & $1.01(0.04)$ & $0.99(0.02)$ \\
Mean wrist velocity $(\mathrm{mm} / \mathrm{s})$ & $308.33(9.26)$ & $319.18(12.38)$ & $309.59(8.83)$ \\
Wrist trajectory $(\mathrm{mm})$ & $310.89(8.03)$ & $314.27(11.91)$ & $301.08(9.18)$ \\
Peak height of wrist $(\mathrm{mm})$ & $99.20(5.74)$ & $101.31(9.93)$ & $90.55(6.47)$ \\
Peak grip aperture $(\mathrm{mm})^{*}$ & $86.50(1.60)$ & $84.06(1.60)$ & $85.61(1.71)$ \\
Lift-to-place phase & & & $1.30(0.15)$ \\
Phase duration $(\mathrm{s})^{*}$ & $1.67(0.17)$ & $1.33(0.14)$ & $411.60(29.78)$ \\
Mean wrist velocity $(\mathrm{mm} / \mathrm{s})^{*}$ & $368.33(30.49)$ & $412.28(32.15)$ & $471.53(23.17)$ \\
Wrist trajectory $(\mathrm{mm})^{*}$ & $522.36(20.73)$ & $484.13(26.46)$ & $260.05(8.68)$ \\
\hline Peak height of wrist $(\mathrm{mm})^{*}$ & $287.60(9.62)$ & $270.23(8.31)$ & \\
\hline
\end{tabular}

${ }^{a}$ Values are mean (SE). Infant vs. Uninformed Adult ${ }^{*}: p<0.05$.

were significantly higher in the Infant condition than in the Uninformed Adult condition $(p<0.05)$.

There was no significant difference in any of the kinematic parameters between the Uninformed Adult and Informed Adult conditions.

\subsection{Mothers' Looking Time}

We investigated the proportions of mothers' total looking time by target in each of the two phases (Table 3). A significant main effect of gaze target was observed in both phases (reach-to-grasp phase: $F(2,28)=200.25, p<0.01, \eta_{p}^{2}=0.94$; lift-to-place phase: $\left.F(2,28)=20.53, p<0.01, \eta_{p}^{2}=0.60\right)$. In addition, there were significant interactions between condition and gaze target in both phases (reach-to-grasp phase: $F(4,56)=7.27, p<0.01, \eta_{p}^{2}=0.34$; lift-to-place phase: $\left.F(4,56)=21.63, p<0.01, \eta_{\mathrm{p}}^{2}=0.61\right)$.

In the reach-to-grasp phase, we found that in all conditions, mothers looked longest at the ball, followed by the observer, and they looked least at the rocket $(p s<0.05)$. In addition, they looked significantly longer at the observer in the Infant than in the Uninformed Adult condition $(p<0.05)$.

In the lift-to-place phase, mothers looked significantly longer at the observer and at the rocket than at the ball in the Infant and Uninformed Adult conditions $(p s<0.05)$. Additionally, in the Informed Adult condition, they looked significantly longer at the rocket than at the observer and the ball ( $p s<0.05)$. They also looked significantly longer at the observer and significantly less at the ball and the rocket ( $p s<0.05)$ in the Infant condition than in the Uninformed Adult condition. Finally, they looked significantly longer at the rocket in the Informed Adult than in the Uninformed Adult condition $(p<0.05)$. 
Table 3. Mean percentage of mothers' total looking time (with standard errors) at each type of target during the reach-to-grasp and lift-to-place phases ${ }^{\mathrm{a}}$.

\begin{tabular}{cccc}
\hline & Infant & $\begin{array}{c}\text { Uninformed } \\
\text { Adult }\end{array}$ & $\begin{array}{c}\text { Informed } \\
\text { Adult }\end{array}$ \\
\hline $\begin{array}{c}\text { Reach-to-grasp phase } \\
\text { Observer (\%) }\end{array}$ & $36.75(4.90)$ & $23.90(4.48)$ & $19.44(4.77)$ \\
Ball (\%) & $62.38(4.89)$ & $73.40(4.21)$ & $78.39(4.55)$ \\
Rocket (\%) & $0.05(0.05)$ & $0.94(0.61)$ & $1.55(0.97)$ \\
Lift-to-place phase & & & \\
Observer (\%) & $55.71(6.77)$ & $36.30(7.54)$ & $25.75(5.74)$ \\
Ball (\%) & $3.84(1.93)$ & $8.89(2.48)$ & $9.64(3.45)$ \\
Rocket (\%) & $38.54(4.99)$ & $51.16(6.94)$ & $61.66(5.19)$ \\
\hline
\end{tabular}

${ }^{a}$ Values are mean (SE).

In summary, these results indicate that mothers' looking times conformed to the same patterns across all conditions in the in the reach-to-grasp phase. However, their looking times differed across conditions in the lift-to-place phase. In both phases, mother looked significantly longer at the observer in the Infant condition than in the Uninformed Adult condition.

\section{Discussion}

The aim of the present study was twofold: first, to investigate whether mothers exaggerate their finger movements when interacting with their infants; and second, to investigate whether a lack of knowledge about the demonstrated manipulation in an adult observer causes characteristics of infant-directed action to emerge. Analysis of the kinematics of mothers' movements showed that demonstrations lasted longer in the Infant condition than in the Uninformed Adult condition and that mothers exaggerated the size of their fine-grained finger movements, as well as their wrist movements, in the Infant condition. However, there was no significant difference in the kinematics parameters relating to mothers' movements between the Informed and Uninformed Adult conditions.

The total duration of the demonstration, the duration of the lift-to-place phase, and the length of wrist trajectories during this phase all exhibited a significant increase in the Infant condition compared to the Uninformed Adult condition. These findings were the same as those of previous studies of infant-directed action (e.g., Brand et al., 2002; Rohlfing et al., 2006). In addition, peak grip aperture was significantly larger in the Infant condition than in the Uninformed Adult condition during the reach-to-grasp phase. This indicates that mothers opened their fingers more widely while reaching for the ball when demonstrating for their infants. Previous research on object manipulation in adult-adult interaction has shown that not only wrist movements but also finger movements are sensitive to the social context of an interaction (Becchio et al., 2008a, 2008b; Vesper et al., 2016). Furthermore, a study using a face-to-face joint grasping task 
has demonstrated that the "leader" exaggerates their peak grip aperture to inform the "follower" about their intended grasp location (Sacheli et al., 2012). On this basis, it was hypothesized that the mother might open her fingers more widely with the aim of providing information about where she intended to grasp the ball to her infant.

Analysis of mothers' gaze behavior showed that they looked significantly longer at the observer in the Infant condition than in the Uninformed Adult condition during both the reach-to-grasp and the lift-to-place phases. A previous study on mother-infant interaction shows that mothers shake and tap an object to attract their infant's attention while gazing at the infant's face (Deák et al., 2014). Therefore, a mother's finger movements might be exaggerated in order to attract her infant's attention, not only while holding the object but even while initially reaching for it, while the mother monitors her infant.

Furthermore, we hypothesized that the characteristics of infant-directed action would emerge when mothers demonstrated how to manipulate an object to an adult who did not know what they were doing. To test this, we compared the kinematics of mothers' movements between the Uninformed Adult and Informed Adult conditions. There was no difference between these conditions in terms of the kinematics of the mothers' movements or the proportion of time spent looking at the observer. It is possible that the characteristics of infant-directed action were not seen in the Uninformed Adult condition because the effect on the mother's demonstration of an adult observer's knowledge, or lack thereof, was small compared to the effect of the infant's characteristics.

A possible reason for this is that the mothers may not have directed their attention to the observer when demonstrating the use of the object to an adult partner, unlike when demonstrating it to their infant. Previous studies show that adults tend to look more at infants, since infants are immature and their attention is scattered compared with that of adults (Glocker et al., 2009; Lorentz, 1965). Additionally, when demonstrating the use of objects to infants, the proportion of time that mothers spend looking at the infant varies with the age of the infant (Brand, Hollenbeck, \& Kominsky, 2013). This suggests that differences in mothers' gaze behaviors can be attributed to the infant's ability to pay attention and that mothers modify their movements to enhance their infants' attention to their actions. Infants' ability to attend is immature compared with that of adults, and they are slow to shift their attention (Butcher, Kalverboer, \& Geuze, 2000; Triesch, Teuscher, Deák, \& Carlson, 2006). In our study, although mothers looked significantly longer at the observer in the Infant condition than in the Uninformed Adult condition, there was no difference in this variable between the Uninformed Adult and Informed Adult conditions. Therefore, it seems that mothers were not trying to attract the attention of the uninformed adult, making it unlikely that the characteristics of infant-directed action would be observed.

The observer's problem-solving abilities might be another reason for the absence of characteristics of infant-directed action when mothers were demon- 
strating to an adult observer. The results of our preliminary test indicated that no adults could quickly figure out how manipulate the toy in the same way demonstrated by the mothers without instruction. A previous study on gesture shows that when a demonstrator feels that their partner has not understood an instruction well, they used more gestures to strengthen communication in an adult-adult interaction (LaFrance \& Mayo, 1978). However, compared with infants, who do not have much experience in various contexts and need adult support, adults have extensive experiences. Thus, the mothers may have thought that the adult observers would be able to manipulate the toy without instruction, meaning that they did not modify the demonstration between the informed and uninformed adults.

A previous study shows that mothers modify their actions depending on their infants' level of achievement in a task (Fukuyama et al., 2015). If this study had examined a condition in which the observer was an infant who was already familiar with the toy, the results of this condition might have been the same as those of conditions involving adults in this study. Further research should address differences between mothers' demonstrations to an infant who is unfamiliar with a toy and an infant who is familiar with it.

\section{Conclusion}

In conclusion, our results suggest that mothers' wrist movements become exaggerated during demonstrations to their infants, and the same is also true of the fine movements of their fingers. However, when mothers demonstrate the use of an object to an adult partner, the observer's knowledge of the use of the object does not affect the characteristics of infant-directed action. Therefore, we suggest that infant-directed action is not caused by the observer's lack of knowledge, but rather by the infant's characteristics (e.g., physical appearance and developing ability to sustained attention). Overall, the current study shows that it is necessary to analyze detailed data, such as finger movements, to examine the mechanisms involved in mothers' adjustments of their motions while demonstrating object manipulation to their infants in everyday life.

\section{Acknowledgements}

We are especially grateful to the families who participated in this work. This research was funded by CREST, JST, and Grant-in-Aid for JSPS Fellows (16J09229 and 15J09751).

\section{Conflicts of Interest}

The authors declare that the research was conducted in the absence of any commercial of financial relationships that could be construed as a potential conflict of interest.

\section{References}

Becchio, C., Sartori, L., Bulgheroni, M., \& Castiello, U. (2008a). Both Your Intention and 
Mine Are Reflected in the Kinematics of my Reach-to-Grasp Movement. Cognition, 106, 894-912. https://doi.org/10.1016/j.cognition.2007.05.004

Becchio, C., Sartori, L., Bulgheroni, M., \& Castiello, U. (2008b). The Case of Dr. Jekyll and Mr. Hyde: A Kinematic Study on Social Intention. Consciousness and Cognition, 17, 557-564. https://doi.org/10.1016/j.concog.2007.03.003

Brand, R. J., \& Shallcross, W. L. (2008). Infants Prefer Motionese to Adult-Directed Action. Developmental Science, 11, 853-861. https://doi.org/10.1111/j.1467-7687.2008.00734.x

Brand, R. J., Baldwin, D. A., \& Ashburn, L. A. (2002). Evidence for "Motionese": Modifications in Mothers' Infant-Directed Action. Developmental Science, 5, 72-83. https://doi.org/10.1111/1467-7687.00211

Brand, R. J., Hollenbeck, E., \& Kominsky, J. (2013). Mothers' Infant-Directed Gaze during Object Demonstration Highlights Action Boundaries and Goals. Transactions on Autonomous Mental Development, 5, 192-201. https://doi.org/10.1109/TAMD.2013.2273057

Butcher, P. R., Kalverboer, A. F., \& Geuze, R. H. (2000). Infants' Shifts of Gaze from a Central to a Peripheral Stimulus: A Longitudinal Study of Development between 6 and 26 Weeks. Infant Behavior Development, 23, 3-21. https://doi.org/10.1016/S0163-6383(00)00031-X

Deák, G. O., Krasno, A. M., Triesch, J., Lewis, J., \& Sepeta, L. (2014). Watch the Hands: Infants Can Learn to Follow Gaze by Seeing Adults Manipulate Objects. Developmental Science, 17, 270-281. https://doi.org/10.1111/desc.12122

Fukuyama, H., Qin, S. B., Kanakogi, Y., Nagai, Y., Asada, M., \& Myowa-Yamakoshi, M. (2015). Infant's Action Skill Dynamically Modulates Parental Action Demonstration in the Dyadic Interaction. Developmental Science, 18, 1006-1013. https://doi.org/10.1111/desc. 12270

Furuyama, N. (2000). Gestural Interaction between the Instructor and the Learner in Origami Instruction. In D. McNeill (Ed.), Language and Gesture (pp.99-117). Cambridge: Cambridge University Press.

Glocker, M. L., Langleben, D. D., Ruparel, K., Loughead, J. W., Gur, R. C., \& Sacheser N. (2009). Baby Schema in Infant Faces Induces Cuteness Perception and Motivation for Caretaking in Adults. Ethology, 115, 257-263. https://doi.org/10.1111/j.1439-0310.2008.01603.x

Hesse, C., \& Deubel, H. (2010). Advance Planning in Sequential Pick-and-Place Tasks. Journal of Neurophysiology, 104, 508-516. https://doi.org/10.1152/jn.00097.2010

Koterba, E. A., \& Iverson, J. M. (2009). Investigating Motionese: The Effect of Infant-Directed Action on Infants' Attention and Object Exploration. Infant Behavioral Development, 32, 437-444. https://doi.org/10.1016/j.infbeh.2009.07.003

LaFrance, M., \& Mayo, C. (1978). Moving Bodies: Nonverbal Communication in Social Relationships. Monterey, CA: Brooks/Cole.

Lausberg, H., \& Sloetjes, H. (2009). Coding Gestural Behavior with the NEUROGES-ELAN System. Behavioral Research Methods, 41, 841-849. https://doi.org/10.3758/BRM.41.3.841

Lorentz, K. (1965). Evolution and Modification of Behavior. Chicago, IL: University of Chicago Press.

Nagai, Y., Nakatani, A., \& Asada, M. (2010). How a Robot's Attention Shapes the Way People Teach. In B. Johansson, E. Sahinand, \& C. Balkenius (Eds.), Proceedings of the 10th International Conference on Epigenetic Robotics (pp. 81-88). Lund: Lund Univer- 
sity.

Oldfield, R. C. (1971). The Assessment and Analysis of Handedness: The Edinburgh Inventory. Neuropsychologia, 9, 97-113. https://doi.org/10.1016/0028-3932(71)90067-4

Rohlfing, K. J., Fritsch, J., Wrede, B., \& Jungmann, T. (2006). How Can Multimodal Cues from Child-Directed Interaction Reduce Learning Complexity in Robots? Advanced Robotics, 20, 1183-1199. https://doi.org/10.1163/156855306778522532

Sacheli, L. M., Candidi, M., Pavone, E. F., Tidoni, E., \& Aglioti, S. M. (2012). And Yet They Act Together: Interpersonal Perception Modulates Visuo-Motor Interference and Mutual Adjustments During a Joint-Grasping Task. PLoS ONE, 7, e50223. https://doi.org/10.1371/journal.pone.0050223

Sartori, L., Becchio, C., Bara, B. G., \& Castiello, U. (2009). Does the Intention to Communicate Affect Action Kinematics? Consciousness and Cognition, 18, 766-772. https://doi.org/10.1016/j.concog.2009.06.004

Streeck, J. (1994). Gesture as Communication II: The Audience as Co-Author. Research on Language and Social Interaction, 27, 239-267. https://doi.org/10.1207/s15327973rlsi2703_5

Triesch, J., Teuscher, C., Deák, G. O., \& Carlson, E. (2006). Gaze Following: Why (Not) Learn It? Developmental Science, 9, 125-147. https://doi.org/10.1111/j.1467-7687.2006.00470.x

Uther, M., Knoll, M. A., \& Burnham, D. (2007). Do You Speak E-NG-L-I-SH? A Comparison of Foreigner- and Infant-Directed Speech. Speech Communication, 49, 2-7. https://doi.org/10.1016/j.specom.2006.10.003

Vesper, C., Schmitz, L., Safra, L., Sebanz, N., \& Knoblich, G. (2016). The Role of Shared Visual Information for Joint Action Coordination. Cognition, 153, 118-123. https://doi.org/10.1016/j.cognition.2016.05.002

Williamson, R. A., \& Brand, R. J. (2014). Child-Directed Action Promotes 2-Year-Olds' Imitation. Journal of Experimental Child Psychology, 118, 119-126. https://doi.org/10.1016/j.jecp.2013.08.005

Wood, D., Bruner, J. S., \& Ross, G. (1976). The Role of Tutoring in Problem Solving. Journal of Child Psychology and Psychiatry, 17, 89-100. https://doi.org/10.1111/j.1469-7610.1976.tb00381.x 


\section{Supplementary Material}

Table A1. Results of $t$ tests comparing the parameters describing the kinematics of mothers' movements across conditions ${ }^{\mathrm{a}}$.

\begin{tabular}{|c|c|c|c|c|c|c|}
\hline & \multicolumn{3}{|c|}{$\begin{array}{c}\text { Infant vs. } \\
\text { Uninformed Adult }\end{array}$} & \multicolumn{3}{|c|}{$\begin{array}{l}\text { Uninformed Adult vs. } \\
\text { Informed Adult }\end{array}$} \\
\hline & $\begin{array}{c}t \text {-value } \\
(\mathrm{df}=14)\end{array}$ & $p$-value & Cohen's $d$ & $\begin{array}{c}t \text {-value } \\
(\mathrm{df}=14)\end{array}$ & $p$-value & Cohen's $d$ \\
\hline Demonstration duration (s) & 4.73 & * & 0.69 & 0.55 & n.s. & 0.03 \\
\hline \multicolumn{7}{|l|}{ Reach-to-grasp phase } \\
\hline Phase duration (s) & 0.48 & n.s. & 0.16 & 0.92 & n.s. & 0.16 \\
\hline Mean wrist velocity $(\mathrm{mm} / \mathrm{s})$ & -1.49 & n.s. & 0.26 & 1.48 & n.s. & 0.23 \\
\hline Wrist trajectory $(\mathrm{mm})$ & -0.43 & n.s. & 0.09 & 1.65 & n.s. & 0.32 \\
\hline Peak height of wrist (mm) & -0.31 & n.s. & 0.07 & 1.57 & n.s. & 0.33 \\
\hline Peak grip aperture (mm) & 2.39 & * & 0.39 & -2.31 & n.s. & 0.23 \\
\hline \multicolumn{7}{|l|}{ Lift-to-place phase } \\
\hline Phase duration (s) & 3.82 & * & 0.60 & 0.37 & n.s. & 0.04 \\
\hline Mean wrist velocity $(\mathrm{mm} / \mathrm{s})$ & -3.74 & * & 0.36 & 0.06 & n.s. & 0.01 \\
\hline Wrist trajectory $(\mathrm{mm})$ & 2.43 & * & 0.46 & 1.04 & n.s. & 0.09 \\
\hline Peak height of wrist (mm) & 4.17 & * & 0.50 & 2.46 & n.s. & 0.31 \\
\hline
\end{tabular}

${ }^{a *}: p<0.05$.

Table A2. Results of $t$ tests comparing mothers' looking times for each type of target in each condition ${ }^{\mathrm{a}}$.

\begin{tabular}{|c|c|c|c|c|c|c|c|c|c|}
\hline & \multicolumn{3}{|c|}{ Infant } & \multicolumn{3}{|c|}{ Uninformed Adult } & \multicolumn{3}{|c|}{ Informed Adult } \\
\hline & $\begin{array}{c}t \text {-value } \\
(\mathrm{df}=14)\end{array}$ & $p$-value & Cohen's $d$ & $\begin{array}{c}t \text {-value } \\
(\mathrm{df}=14)\end{array}$ & $p$-value & Cohen's $d$ & $\begin{array}{c}t \text {-value } \\
(\mathrm{df}=14)\end{array}$ & $p$-value & Cohen's $d$ \\
\hline \multicolumn{10}{|l|}{ Reach-to-grasp phase } \\
\hline Observer vs. Ball & -3.07 & * & 1.58 & -6.67 & * & 3.38 & -7.16 & * & 3.65 \\
\hline Observer vs. Rocket & 13.53 & * & 5.14 & 7.84 & * & 3.30 & 5.64 & * & 2.33 \\
\hline Ball vs. Rocket & 20.89 & * & 7.42 & 23.78 & * & 8.05 & 19.68 & * & \\
\hline \multicolumn{10}{|l|}{ Lift-to-place phase } \\
\hline Observer vs. Ball & 7.03 & * & 3.34 & 3.15 & * & 1.26 & 2.06 & n.s. & 0.90 \\
\hline Observer vs. Rocket & 1.56 & n.s. & 0.78 & -1.42 & n.s. & 0.71 & -3.82 & * & 1.83 \\
\hline Ball vs. Rocket & -14.95 & * & 3.26 & -6.73 & * & 2.56 & -8.71 & * & 3.39 \\
\hline
\end{tabular}

${ }^{\mathrm{a} *}: p<0.05$.

Table A3. Results of $t$ tests comparing mothers' looking times for each type of target in each condition ${ }^{\mathrm{a}}$.

\begin{tabular}{|c|c|c|c|c|c|c|}
\hline & \multicolumn{3}{|c|}{$\begin{array}{c}\text { Infant vs. } \\
\text { Uninformed Adult }\end{array}$} & \multicolumn{3}{|c|}{$\begin{array}{l}\text { Uninformed Adult vs. } \\
\text { Informed Adult }\end{array}$} \\
\hline & $\begin{array}{c}t \text {-value } \\
(\mathrm{df}=14)\end{array}$ & $p$-value & Cohen's $d$ & $\begin{array}{c}t \text {-value } \\
(\mathrm{df}=14)\end{array}$ & $p$-value & Cohen's $d$ \\
\hline Reach-to-grasp phase & & & & & & \\
\hline
\end{tabular}




\section{Continued}

\begin{tabular}{lcccccc}
\hline Observer & 2.80 & $*$ & 0.86 & 1.31 & n.s. & 0.35 \\
Ball & -2.26 & n.s. & 0.75 & -1.50 & n.s. & 0.41 \\
Rocket & -1.76 & n.s. & 0.66 & -0.92 & n.s. & 0.19 \\
Lift-to-place phase & & & & & & \\
Observer & 4.70 & $*$ & 0.81 & 2.08 & n.s. & 0.38 \\
Ball & -2.99 & $*$ & 0.84 & 0.17 & n.s. & 0.03 \\
Rocket & -3.65 & $*$ & 0.65 & -2.59 & $*$ & 0.48 \\
\hline
\end{tabular}

${ }^{\mathrm{a} *}: p<0.05$.

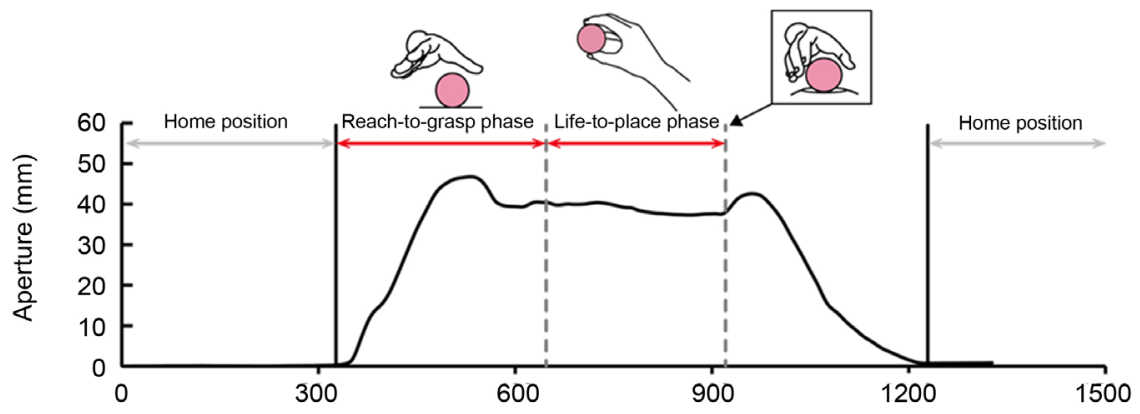

(a)

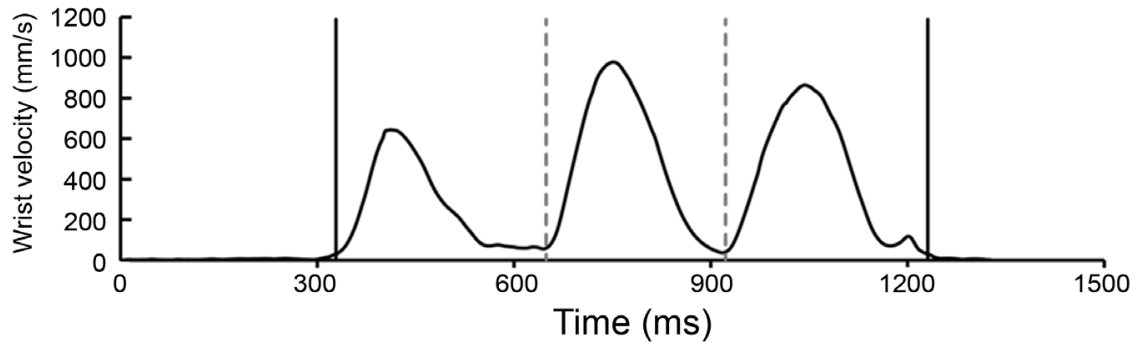

(b)

Figure A1. Example of typical grip aperture profile (a) and wrist velocity profile (b) during manipulation of the ball. The onset and end of the ball manipulation period were determined as follows. The first frame in which the wrist velocity exceeded a threshold of 25 $\mathrm{mm} / \mathrm{s}$ after the mother's hand moved away from the home position was considered the onset of the ball manipulation. The frame in which the wrist velocity fell below a threshold of $25 \mathrm{~mm} / \mathrm{s}$ after the mother had put the ball into the rocket and then returned her hand to the home position was considered the end of the ball manipulation. The onset and end of each action phase were defined using a spatial criterion and the velocity of the wrist, based on Hesse and Deubel's (2010) method. The onset of the reach-to-grasp phase coincided with the onset of ball manipulation; the end of this phase was the frame in which the wrist velocity first reached its minimum. This trough in wrist velocity indicated that the mother's hand had reached the ball. The onset of the lift-to-place phase was the next frame following the end of the reach-to-grasp phase, and the end of this phase occurred when the wrist velocity reached its minimum for the second time. Note: Figure A1 denotes an example of typical scene in this study. Source: This figure is our original creation. 